\title{
A Nonlinear Optimal Control Design using Narrowband Perturbation Feedback for Magnetostrictive Actuators
}

\author{
William S. Oates ${ }^{1}$, Rick Zrostlik ${ }^{2}$, Scott Eichhorn ${ }^{2}$, Ralph Smith ${ }^{3}$ \\ ${ }^{1}$ Florida Center for Advanced Aero Propulsion (FCAAP), Department of Mechanical Engineering, \\ Florida State University, Tallahassee, FL 32306-2870 \\ ${ }^{2}$ Etrema Products, Inc., Ames, IA 50010 \\ ${ }^{3}$ Department of Mathematics, North Carolina State University, Raleigh, NC 27695
}

\begin{abstract}
Nonlinear optimal and narrowband feedback control designs are developed and experimentally implemented on a magnetostrictive Terfenol-D actuator. The nonlinear optimal control design incorporates a nonlinear and hysteretic ferromagnetic homogenized energy model within an optimal control formulation to reduce displacement tracking errors and increase bandwidth. Improvements in robustness in the steady-state regime are achieved by utilizing narrowband feedback. A narrowband filter is implemented by treating the nonlinear and hysteretic magnetostrictive constitutive behavior as higher-order harmonic disturbances which are mitigated by tuning the narrowband filter to penalize these harmonics for displacement tracking control problems. The control designs are then combined into a hybrid optimal controller with perturbation narrowband feedback. Both transient and steady-state tracking control is assessed to illustrate performance attributes in different operating regimes. Narrowband perturbation feedback is shown to mitigate errors in the steady-state operating regime, while nonlinear optimal control provides enhanced tracking control in the transient regime. The hybrid control design is relevant to a broad number of smart material actuators that exhibit nonlinear and hysteretic fieldcoupled constitutive behavior.
\end{abstract}

\section{Introduction}

Magnetostrictive materials provide an important component for many adaptive structure applications due to their magneto-mechanical properties, broad bandwidth capabilities, and reliability. These materials have been extensively utilized in a number of commercial and military applications such as sonar transducers, precision machining, and force feedback surgical training tools (Smith 2005). These materials exhibit broadband micro to nanopositioning characteristics. For example, piezoelectric actuators are often used in nanopositioning of atomic force microscope stages. The actuator shown in Figure 1 is a relatively long actuator which can provide displacements on the order of 100's of microns using a mechanical amplification 
structure (not shown) to amplify the displacement by an order of magnitude. Nanoscale resolution can be achieved by eliminating the displacement amplification mechanism and reducing the length of the actuator. These materials also produce nonlinear and hysteretic fieldcoupled material behavior when driven at medium to large field amplitudes. This behavior is primarily due to the reorientation of microscale ferromagnetic domain structures which can produce significant departures from linearity. Ferromagnetic behavior strongly depends on the mechanical preload, bias field, loading rate, loading direction, etc., and can complicate the actuator design and control law required to meet stringent performance criteria. A typical open loop response for a magnetostrictive Terfenol-D rod operating in the nonlinear and hysteretic regime is illustrated in Figure 1. This actuator consists of a wound wire solenoid surrounding the magnetostrictive Terfenol-D rod which is preloaded with a spring-compression bolt assembly. Current applied to the solenoid generates magnetic fields along the rod and subsequently nonlinear and hysteretic magnetostriction at medium to high drive levels.

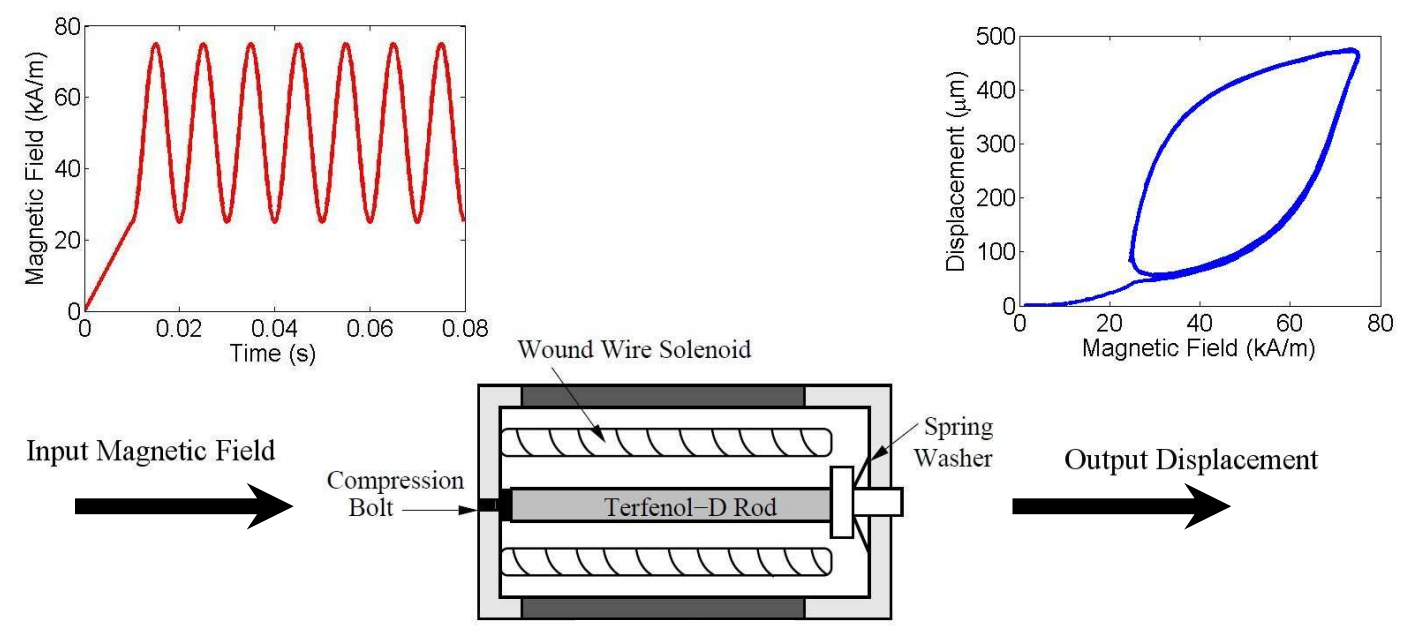

Figure 1: Typical open loop response of a Terfenol-D actuator operating in the nonlinear and hysteretic regime. A sinusoidal voltage input is applied to a wound wire solenoid surrounding a magnetostrictive Terfenol-D rod. This results in a distorted output displacement due to ferromagnetic domain structure evolution and magnetostriction.

Nonlinear control designs are often necessary to compensate for the magnetostrictive nonlinearities. Such control designs are also useful for a broader class of active materials such as ferroelectric materials and shape memory alloys (Smith 2005). Tracking control and disturbance rejection of nonlinear materials and structures has been studied extensively to compensate for 
external vibration loads or internal disturbances (Clarke 1987; Croft 2001; Ge 1996; Ikhouane 2007; Iyer 2009; Iyer 2005; Janocha 2000; Janocha 2008; Mracek 1998; Natale 2001; Oates 2009a; Sievers 1992; Tan 2004). These control designs can be broadly classified as inverse compensators, model predictive control, adaptive control, or direct nonlinear control. Inverse compensators and model predictive control designs have the advantage of tracking unknown reference displacements or attenuating vibration or noise. These control designs are normally limited to low to moderate frequencies due to computational limitations of inverting the material model in real time (Henson 1998; Tan 2004). This is a particular challenge for hysteretic actuators. Adaptive control methods have shown to be effective in systems with significant uncertainty where model predictions are highly complex such as high speed flow (Tian 2006). In contrast, nonlinear control designs avoid computational limitations required to invert the constitutive relations, but typically require computing a nonlinear open loop control signal offline (Oates 2009a; Oates 2009b). This limits the control design to applications where the reference signal or disturbance is known in advance, but provides superior bandwidth and reduced tracking errors since the nonlinear constitutive behavior is directly compensated without feedback. Without feedback, this approach lacks robustness; therefore, perturbation feedback is often implemented in combination with the open loop signal to compensate for uncertainties in the model or unknown disturbances.

Proportional Integral (PI) perturbation feedback has been coupled with this nonlinear control design to improve robustness to operating uncertainties; see (Oates 2009a). The experiments presented in (Oates 2009a) used a Terfenol-D actuator that contained a permanent magnet for operation about a biased magnetization point. Biased magnetization allows the actuator to produce bi-directional motion when subjected to a bi-directional input current. Also, biased magnetization can allow operation in a magnetostrictive region that result in reduced overall nonlinearities. Whereas reasonable nonlinear tracking control was achieved in the presence of minor loop hysteresis, improved robustness over a broader operating regime is desired. It should be further noted that these experiments were conducted on periodic sinusoidal reference displacements which presented significant challenges in extending the tracking control problem to more general, non-periodic reference displacements that may contain abrupt changes in the displacement signal. The proportional-integral (PI) perturbation feedback design was found to be non-robust to non-sinusoidal displacement tracking. Therefore, more robust perturbation feedback designs are of interest to utilize the broad bandwidth capabilities for more general displacement reference tracking problems. 
Narrowband control often introduces a cost function to penalize vibration disturbances or acoustic emission at specified frequencies; see (Hall 1992; Sievers 1992) for a review. One type of narrowband control, higher harmonic control, is well known for its robustness in controlling complex vibration in helicopters without complete knowledge of the helicopter dynamics (Cheng 2006; Lovera 2003). This robustness is achieved by weighting control gains at the disturbance load frequencies such as the harmonics of a rotorblade's angular velocity. Interactions between the plant dynamics and the narrowband filter are therefore normally avoided since the forced vibration loads are typically much lower than the resonant modes of the structure.

The narrowband control design utilizes optimal control theory to obtain a feedback control design of an augmented plant that includes a number of narrowband harmonics at specified frequencies. This is typically formulated at steady state which leads to a suboptimal control design or infinite horizon problem (Bay 1999). An algebraic Ricatti equation is solved to determine the feedback control gains. This approach can provide improved steady state vibration attenuation, but has limited transient performance. For example, the settling time is inversely related to the feedback control gain. As this gain is increased, the bandwidth increases; however, closed loop poles associated with the filter may approach the actuator poles, perhaps in an unstable manner. This may also occur in the nonlinear magnetostrictive actuator; therefore, improved robustness may be obtained by coupling narrowband perturbation feedback with nonlinear optimal control to enhance both steady-state tracking errors and transient performance, respectively. This effectively "linearizes" the actuator so that feedback control signals that compensate for nonlinear constitutive behavior are reduced. The narrowband feedback is then utilized to compensate for smaller tracking errors that may be due to model uncertainties or external electrical or mechanical disturbances.

In the analysis presented here, a narrowband filter and integral control are both compared and combined with a nonlinear optimal control design for tracking control problems. The narrowband feedback design is formulated to compensate for internal periodic disturbances related to magnetostrictive material nonlinearities and hysteresis but can also accommodate external disturbances or be applied to similar active material actuators. The nonlinear optimal control design is based on a model and experiments described in (Oates 2009a; Oates 2009b). In the analysis presented here, tracking control is achieved in a significantly larger nonlinear and hysteretic operating regime. Lastly, the nonlinear optimal control design is coupled with the narrowband feedback control as a hybrid control design with narrowband-integral perturbation feedback. In Section II, nonlinear and hysteretic modeling of the magnetostrictive actuator is presented which includes a brief description of the magnetostrictive homogenized energy model. 
This is followed by open loop characterization of the magnetostrictive actuator and comparison of the model in Section III. The model-based nonlinear optimal controller and narrowband feedback designs are then developed and compared to control experiments in Sections III and IV.

\section{Homogenized Energy Modeling of Magnetostrictive Materials}

Homogenized energy modeling has been used extensively to predict nonlinear and hysteretic constitutive behavior of ferroelectric, ferromagnetic, and shape memory alloy materials (Smith 2005). A brief summary of the model is given here to illustrate the approach used to predict the constitutive behavior and to motivate how the model is integrated into the nonlinear optimal control design.

The homogenized energy model couples statistical mechanical tenets with continuum mechanics by introducing an underlying distribution of certain material parameters that govern local switching of ferromagnetic domain structures. Mean-field approximations are used to predict the ferromagnetic behavior by super-imposing the applied field with distributions of internal interaction fields which together are denoted as a stochastic, effective field. When the effective field equals a local coercive field, ferromagnetic switching occurs. These local switching events are homogenized based on the probability distributions of the interaction fields and coercive fields.

The model is based on a non-convex energy description at the single crystal length scale

$$
G(M, T)=\Psi(M, T)-\mu_{0} H M
$$

where $\Psi(M, T)$ is a Landau energy function, $\mu_{0}$ is the permeability of free space, $\mathrm{T}$ is the temperature, $H$ is the magnetic field, and $M$ is the magnetization. In the one-dimensional case used for controlling the magnetostrictive actuator, the field, magnetization, and material parameters are considered scalar coefficients or distributed parameters in the direction of loading. Moreover, the one-dimensional model is defined by a double-well Landau energy function with stable positive and negative remanent magnetization.

In most control designs, rate-dependent effects are important and must be incorporated into the control design. Rate-dependent ferromagnetic hysteresis is introduced using the Boltzmann distribution

$$
\mu(G)=C e^{-G V / k T}
$$


where this relation defines the probability $\mu(G)$ of achieving an energy level $G$. The relative thermal energy is $k T / V$ where $V$ is a representative volume element at the microscopic length scale, $k$ is Boltzmann's constant, and the constant $C$ is specified to ensure integration to unity. As the thermal energy increases, the energy barrier required to jump from one energy well to another is reduced which reduces the sharp transition of magnetic variants switching from one state to another.

The single crystal magnetization is defined as

$$
\bar{M}=x_{+}\left\langle M_{+}\right\rangle+x_{-}\left\langle M_{-}\right\rangle
$$

where $x_{+}$is the volume fraction of positive magnetic variants and $x_{-}$is the volume fraction of negative variants and the average local magnetization in the positive and negative direction is denoted by $\left\langle M_{+}\right\rangle$and $\left\langle M_{-}\right\rangle$, respectively. These average values are determined from a balance of the nonconvex energy function in (1) and the probability estimates of thermal energy in (2). A set of differential equations define the time dependent evolution of the internal state based on likelihoods of ferromagnetic switching that accounts for relaxation behavior. Details describing this aspect of the model can be found in (Smith 2006).

The homogenized or macroscopic prediction of magnetization is computed via the relation

$$
[M(H)](t)=\int_{0}^{\infty} \int_{-\infty}^{\infty} v\left(H_{c}, H_{I}\right) \bar{M}(t) d H_{c} d H_{I}
$$

where $v\left(H_{c}, H_{I}\right)$ describes the distribution of the coercive field $H_{c}$ and the interaction field $H_{I}$. These parameters constitute a reduced set of parameters governing material inhomogeneities present in polycrystalline magnetostrictive materials. This may include variations in composition, grain boundary effects, variations in local crystal anisotropy, etc. Log-normal and normal distributions on the coercive field and interaction field, respectively, are often sufficient to estimate the hysteresis. The model fits used in the control design; however, were determined using a general density formulation which was identified using standard stochastic optimization techniques (Kirkpatrick 1983). This approach provides better model fits in the nonlinear regime as previously illustrated in Figure 2. It should be noted that the constitutive model predictions in 
Figure 2 are based on a single set of material parameters that predict minor loop hysteresis at different field amplitudes.

The constitutive relation quantifying magnetostrictive coupling is

$$
\sigma=Y^{M} \varepsilon-a_{1}\left(M(H)-M_{0}\right)-a_{2}\left(M(H)-M_{0}\right)^{2}
$$

where $\sigma$ is uni-axial stress, $Y^{M}$ is the Young's modulus at fixed magnetization, $\varepsilon$ is infinitesimal strain in the loading direction, $a_{1}$ is the piezomagnetic coefficient, $a_{2}$ is the magnetostrictive coefficient, and $M_{0}$ is the remanent magnetization.

The relations in (4) and (5) quantify the macroscopic constitutive behavior of the magnetostrictive rod which is used to define the structural dynamics. Although finite element formulations could be used to obtain spatially dependent stress distributions, the reference frequency under consideration is below mechanical resonance. Therefore, we employ the lumped parameter model

$$
m \ddot{x}+c_{D} \dot{x}+k x=F_{m a g}(H)
$$

where $m, c_{D}$, and $k$ are the effective mass, damping, and stiffness of the magnetostrictive rod, $A$ is the cross-section area, and $\left.F_{\text {mag }}(H)=A \mid a_{1}\left(M(H)-M_{0}\right)+a_{2}\left(M(H)-M_{0}\right)^{2}\right]$.

This can be formulated as a set of first order equations

$$
\begin{aligned}
\dot{\mathbf{x}} & =\mathbf{A} \mathbf{x}+\mathbf{B}(u) \\
y & =\mathbf{C} \mathbf{x}
\end{aligned}
$$

where $\mathbf{x}=\left[\begin{array}{ll}x & \dot{x}\end{array}\right]^{T}$ subject to a set of initial conditions $\mathbf{x}(0)=\mathbf{x}_{0}$. The matrices are defined by

$$
\mathbf{A}=\left[\begin{array}{cc}
0 & -1 \\
-k / m & -c_{D} / m
\end{array}\right], \quad \mathbf{B}=\left[\begin{array}{c}
0 \\
1 / m
\end{array}\right] F_{m a g}(H), \quad \text { and } \quad \mathbf{C}=\left[\begin{array}{ll}
1 & 0
\end{array}\right]
$$

The parameters used in the homogenized energy model and the damped oscillator model described by (7) and (8) are given in Table 1. This model is implemented in the nonlinear control 
design in the following section. Control simulations are given and then compared to experimental results.

Table 1: Homogenized energy parameters used to predict the constitutive behavior of the magnetostrictive actuator. The parameters $\mathrm{M}^{\mathrm{r}}, \eta, \tau, \gamma$ denote the remanent magnetization, inverse susceptibility, time constant, and thermal energy; respectively; see (Smith 2005) for more details.

\begin{tabular}{|l|l|}
\hline $\mathrm{a}_{1}$ & $1.4 \times 10^{-5} \mathrm{~N} /(\mathrm{A} \times \mathrm{m})$ \\
\hline $\mathrm{a}_{2}$ & $6.44 \times 10^{-8} \mathrm{~N} / \mathrm{A}^{2}$ \\
\hline $\mathrm{M}^{\mathrm{r}}$ & $19.3 \mathrm{kA} / \mathrm{m}$ \\
\hline$\eta$ & 4.06 \\
\hline$\tau$ & $5.02 \mathrm{sec}$ \\
\hline$\gamma$ & $0.87 \mathrm{~m} \times \mathrm{s}^{2} / \mathrm{kg}$ \\
\hline $\mathrm{k} / \mathrm{m}$ & $1.7 \times 10^{7}(\mathrm{rad} / \mathrm{sec})^{2}$ \\
\hline $\mathrm{c}_{\mathrm{D}} / \mathrm{m}$ & $9.1 \times 10^{3}(\mathrm{rad} / \mathrm{sec})$ \\
\hline
\end{tabular}

\section{Terfenol-D Actuator and Open Loop Characterization}

A Terfenol-D magnetostrictive actuator is used to validate the nonlinear optimal control design. The design of the actuator is based on the schematic previously illustrated in Figure 1. Open loop characterization is conducted to identify constitutive model parameters used to then determine the nonlinear control input. The data was collected using (AE Techron 7780 linear amplifier, DS1003 dSpace processor board, Matlab V5.2/Simulink V2.2.1, Schaevitz 025MHR LVDT). The experimental results and comparisons with the homogenized energy model, as is summarized in the following section, are illustrated in Figure 2. Additional discussion on the experimental control set-up is given in SectionV. 


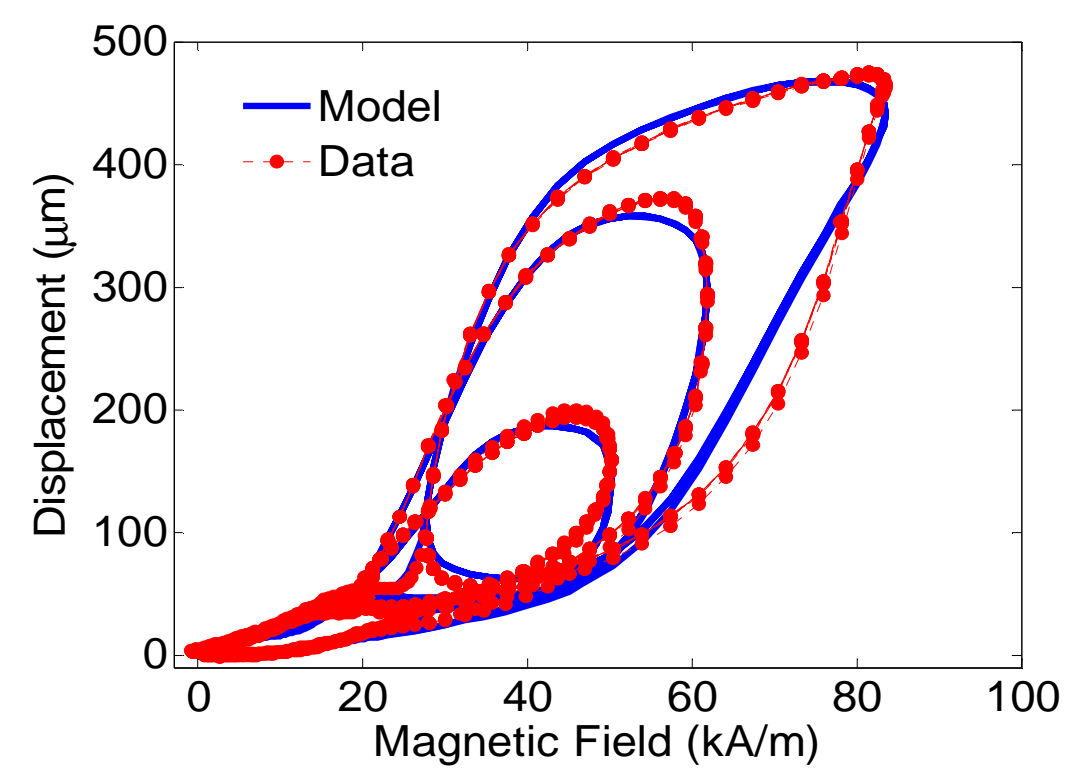

Figure 2: Nonlinear and hysteretic material behavior of the Terfenol-D rod actuator. A sinusoidal input of $100 \mathrm{~Hz}$ was applied to the actuator at multiple field amplitudes.

\section{Control Design}

We summarize here the nonlinear optimal and narrowband control designs. Simulations are then given illustrating tracking control performance for nonlinear optimal control and hybrid nonlinear control with narrowband perturbation feedback. The effect of multiple narrowband harmonics is assessed with and without the nonlinear optimal control input. Simulations are then compared with experimental results Section V.

\section{IV.1 Nonlinear Optimal Tracking Control}

A previously developed nonlinear optimal control design is briefly summarized here; more details can be found in (Oates 2009b). The formulation utilizes optimal control theory where a cost function is defined to penalize tracking errors and the control input (Lewis 1995). Through the utilization of Lagrange multipliers, an unconstrained minimization of the cost function is formulated to determine the nonlinear control input. This requires solving a two-point boundary value problem that is not trivial for large nonlinear and hysteretic models. This issue is avoided by implementing an LU decomposition of the system of equations. A brief summary of this methodology is given as follows.

The cost functional is 


$$
\bar{J}=\frac{1}{2}\left(C x\left(t_{f}\right)-r\left(t_{f}\right)\right)^{T} P\left(C x\left(t_{f}\right)-r\left(t_{f}\right)\right)+\int_{t_{0}}^{t_{f}}\left(H-\lambda^{T}(t) \dot{x}(t)\right) d t
$$

where $r(t)$ is the reference signal, $P$ is the penalty on the final state, $H$ is the Hamiltonian, and $\lambda^{T}(t)$ is a set of Lagrange multipliers.

The Hamiltonian is

$$
H=\frac{1}{2}\left[(\mathbf{C x}(t)-r(t))^{T} Q(\mathbf{C x}(t)-r(t))+u^{T}(t) R u(t)\right]+\lambda^{T}[\mathbf{A x}(t)+[\mathbf{B}(u)](t)]
$$

which penalizes measured displacements and the control input via the parameters $Q$ and $R$, respectively.

The nonlinear control input is determined by minimizing the cost in (10) subject to the constraint of the dynamic system in (7). The resulting optimality system is

$$
\begin{aligned}
& {\left[\begin{array}{l}
\dot{\mathbf{x}}(t) \\
\dot{\lambda}(t)
\end{array}\right]=\left[\begin{array}{c}
\mathbf{A x}(t)+[\mathbf{B}(u)](t) \\
-\mathbf{A}^{\mathrm{T}} \lambda(t)-\mathbf{C}^{\mathrm{T}} \mathbf{Q} \mathbf{C} \mathbf{x}(t)+\mathbf{C}^{\mathrm{T}} \mathbf{Q} r(t)
\end{array}\right]} \\
& \mathbf{x}\left(t_{0}\right)=\mathbf{x}_{0} \\
& \lambda\left(t_{f}\right)=\mathbf{C}^{\mathrm{T}} \mathbf{P}\left(\mathbf{C} \mathbf{x}\left(t_{f}\right)-r\left(t_{f}\right)\right) .
\end{aligned}
$$

The corresponding optimal control input based on the stationary condition on the Hamiltonian is

$$
u^{*}(t)=-R^{-1}\left(\frac{\partial B(u)}{\partial u}\right)^{T} \lambda(t)
$$

The nonlinear system in (12) is solved numerically using a central difference temporal discretization and LU decomposition; see (Oates 2009b) for details. The numerical approach is employed since a simple Ricatti formulation is not possible due to the nonlinear nature of the input operator. The solution of this nonlinear set of equations gives the optimal control input in (13) which is experimentally implemented as a feedforward control signal. Before illustrating performance attributes, the narrowband perturbation feedback control design is summarized. 


\section{IV.2 Narrowband Feedback Control}

The cost function used in the narrowband control design is formulated to penalize tracking errors that are generated from internal periodic disturbances associated with nonlinear and hysteretic magnetostrictive behavior; however, the control design is general and can accommodate external periodic disturbances and nonlinear constitutive behavior of other smart materials such as ferroelectric materials or shape memory alloys. Integral control is included in the feedback loop to reduce static tracking errors. In the case considered here, the magnetostrictive constitutive behavior creates significant internal disturbances that produce higher harmonics. This is demonstrated experimentally in Figure 3(a) for the quasi-static case of $0.5 \mathrm{~Hz}$ where actuator displacement versus input current is plotted. This emphasizes that higher harmonics are influenced by the magnetostrictive behavior and ferromagnetic hysteresis. The actuator displacement for a $100 \mathrm{~Hz}$ sinusoidal input current was also measured in open loop. The fast Fourier transform of these measurements are plotted in Figure 3(b) which clearly illustrate non-negligible higher order harmonics. The narrowband filter is designed to penalize tracking errors at these discrete frequencies.

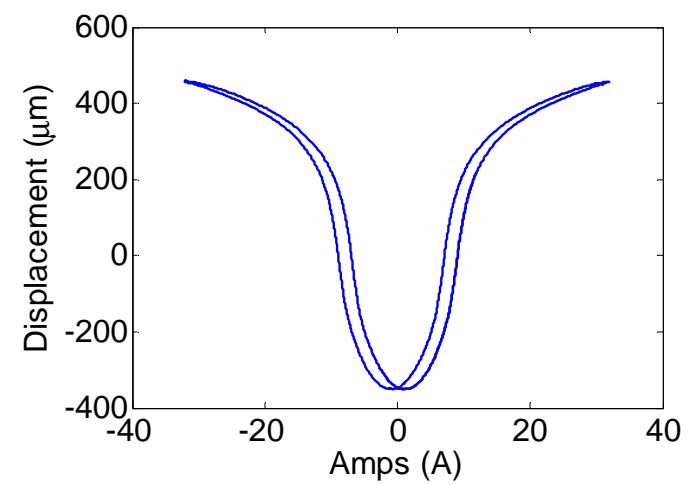

(a)

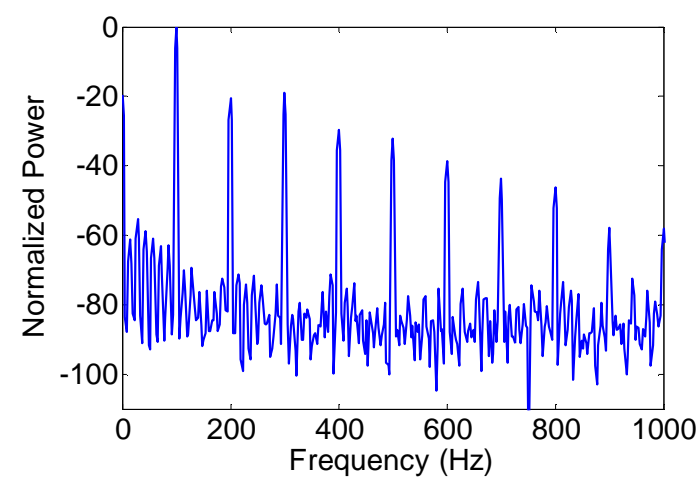

(b)

Figure 3: (a) Quasi-static hysteresis measurements for a $0.5 \mathrm{~Hz}$ sinusoidal current input. (b) An FFT plot of the output displacement for a $100 \mathrm{~Hz}$ input current which illustrates the presence of higher harmonic disturbances.

The control design is formulated as a disturbance model that is coupled to a linearized version of magnetostrictive actuator model. This assumes small perturbations about an operating point. The narrowband filter and linearized actuator relations are

$$
\dot{\mathbf{x}}_{f}=\mathbf{A}_{f} \mathbf{x}_{f}+\mathbf{B}_{f} y
$$




$$
\begin{aligned}
& y_{f}=\mathbf{C}_{f} \mathbf{x}_{f} \\
& \dot{\mathbf{x}}=\mathbf{A x}+\tilde{\mathbf{B}} u \\
& y=\mathbf{C x}
\end{aligned}
$$

where $\widetilde{\mathbf{B}}$ is the linearized input operator of the actuator. The narrowband filter consists of the filter states $\mathbf{x}_{f}$, and second order filter matrices $\mathbf{A}_{f}, \mathbf{B}_{f}, \mathbf{C}_{f}$. The augmented system of equations is

$$
\begin{aligned}
& {\left[\begin{array}{c}
\dot{\mathbf{x}}_{f} \\
\dot{\mathbf{x}}
\end{array}\right]=\left[\begin{array}{cc}
\mathbf{A}_{f} & \mathbf{B}_{f} \mathbf{C} \\
\mathbf{0} & \mathbf{A}
\end{array}\right]\left[\begin{array}{c}
\mathbf{x}_{f} \\
\mathbf{x}
\end{array}\right]+\left[\begin{array}{c}
0 \\
\tilde{\mathbf{B}}
\end{array}\right] u} \\
& y=\left[\begin{array}{ll}
0 & \mathbf{C}
\end{array}\right]\left[\begin{array}{c}
\mathbf{x}_{f} \\
\mathbf{x}
\end{array}\right] .
\end{aligned}
$$

The form of the second-order filter matrices are

$$
\mathbf{A}_{f i}=\left[\begin{array}{cc}
-2 \xi_{i} \omega_{i} & -\omega_{i}^{2} \\
1 & 0
\end{array}\right], \quad \mathbf{B}_{f i}=\left[\begin{array}{l}
1 \\
0
\end{array}\right], \text { and } \quad \mathbf{C}_{f}=\left[\begin{array}{ll}
1 & 0 \\
0 & 1
\end{array}\right]
$$

where $\omega_{i}$ is the resonant frequency and $\xi_{i}$ is a damping parameter for each filter state $i=1$ to $n$. The matrix $\mathbf{A}_{f}$ is composed of a diagonal matrix that includes a number of sub-system matrices $\mathbf{A}_{f i}$ for $i=1$ to $n$ where $n$ defines the number of higher-order harmonics that penalize internal disturbances at each frequency $\omega_{i}$. Similarly, a column matrix with repeated matrix components of $\mathbf{B}_{f i}=\left[\begin{array}{lllll}1 & 0 & \ldots & 1 & 0\end{array}\right]^{T}$ with dimensions $[2 n \times 1]$ is used. The filter is defined to be fully observable by defining the output matrix $\mathbf{C}_{f}$ to be the identity.

Optimal control theory is applied to obtain a sub-optimal control gain for the augmented system in (18). The cost function is

$$
\left.J=\int_{0}^{\infty}\left\{\begin{array}{ll}
\mathbf{x}_{f}^{T} & e
\end{array}\right] \mathbf{Q}_{f}\left[\begin{array}{ll}
\mathbf{x}_{f} & e
\end{array}\right]+u_{N B} R_{f} u_{N B}\right\} d t
$$


where the tracking error is defined by $e=y-r, \mathbf{Q}_{f}$ is a matrix that is used to penalize each defined tracking error harmonic, and $R_{f}$ penalizes the narrowband control input $u_{N B}$.

The feedback control gain is determined by minimizing the cost function in (20) which leads to an algebraic Ricatti equation; see (Bay 1999) for details. The control gain is

$$
u_{N B}=-\left[\begin{array}{ll}
\mathbf{K}_{f} & \mathbf{K}
\end{array} \mathbf{x}_{f}^{T} \quad e\right]
$$

where $\mathbf{K}_{f}$ is the filter gain with dimension [2n×1] and the control gain for the linearized plant is K. Since the penalties on harmonics of the reference frequency are of primary interest, the control gain $\mathbf{K}$ is set to zero and narrowband perturbation feedback is based only on $\mathbf{K}_{f}$. This approach heavily penalizes tracking errors centered at each frequency $\omega_{i}$ that is included in the narrowband filter. The damping parameter $\xi_{i}$ is set to zero to provide the largest disturbance rejection in tracking displacement errors.

In addition to narrowband feedback, an integral control input is included in the perturbation feedback loop in parallel with the narrowband filter. This provides sufficient low frequency gains to minimize static tracking errors. A conventional integral feedback term of the

form $u_{I}=\int K_{I}(y(t)-r(t)) d t$ is introduced in the control loop where $K_{\mathrm{I}}$ is the integral gain, $y(t)$ is the measured displacement, and $r(t)$ is the reference displacement.

In summary, the perturbation feedback control input, including both the narrowband $u_{N B}$ and integral $u_{I}$ control, is coupled with the feedforward open loop control signal $u^{*}$ given by (13) to give the total control input

$$
u=u^{*}+u_{N B}+u_{I}
$$

A comparison of each control signal and the combination of all control input terms are simulated to quantify tracking performance as described in the following section. This is followed by experimental validation in Section V.

\section{IV.3 Simulation Results}


Simulations are given to illustrate differences in tracking control and to provide comparisons with experiments. Open loop nonlinear optimal control simulations are compared to the hybrid nonlinear optimal control design using narrowband-integral perturbation feedback. The results illustrate improved transient response when the nonlinear optimal control is applied in open loop while narrowband perturbation feedback reduces tracking errors that occur in the presence of model uncertainties.

Open loop simulations are first given to illustrate tracking control performance in the nonlinear regime previously illustrated in Figure 2. The open loop simulations shown in Figure 4 illustrate minimal tracking errors in this nonlinear and hysteretic regime. The nonlinear control input directly compensates for the constitutive response shown in Figure 4(b). Whereas excellent tracking is achieved, these results are non-robust since model uncertainties are not considered. Model uncertainties are introduced into the control design using set of modified material parameters. These parameters were obtained by fitting a subset of the data previously shown in Figure 2. The simulated annealing optimization algorithm was used again to calculate a set of material parameters that were only fit to data that contained displacement between $0 \mu \mathrm{m}$ and 350 $\mu \mathrm{m}$. Using the material parameters fit for only this 0-350 $\mu \mathrm{m}$ displacement, open loop tracking errors increased from an RMS error of $0.81 \%$ in Figure 4 to $7.11 \%$ with the modified material parameters; see Figure 5. A fast Fourier transform of the tracking error in the steady state oscillating regime is plotted in Figure 5(b).

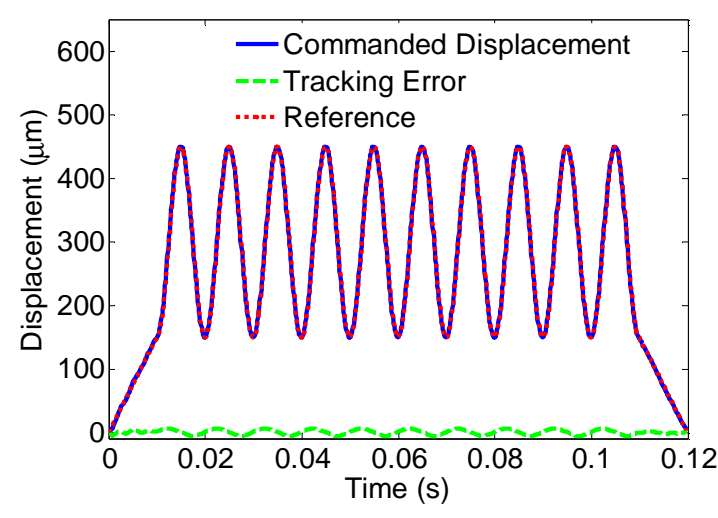

(a)

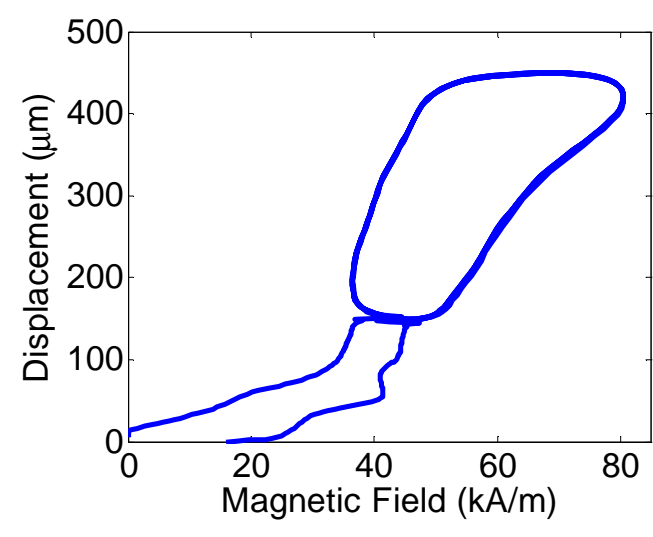

(b)

Figure 4: Nonlinear open loop simulations assuming ideal model parameters in the (a) time domain and (b) phase space show the homogenized energy model prediction. 


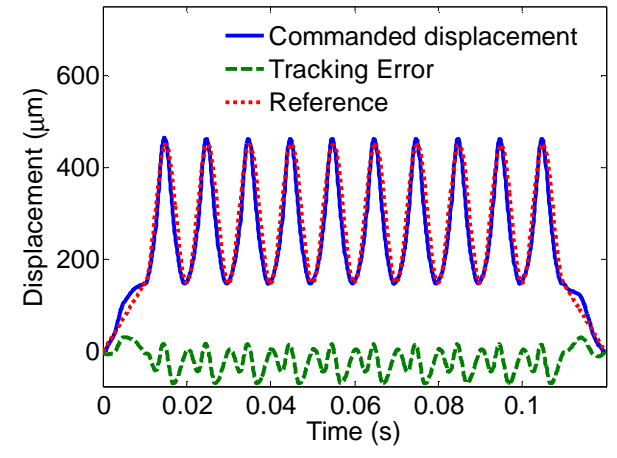

(a)

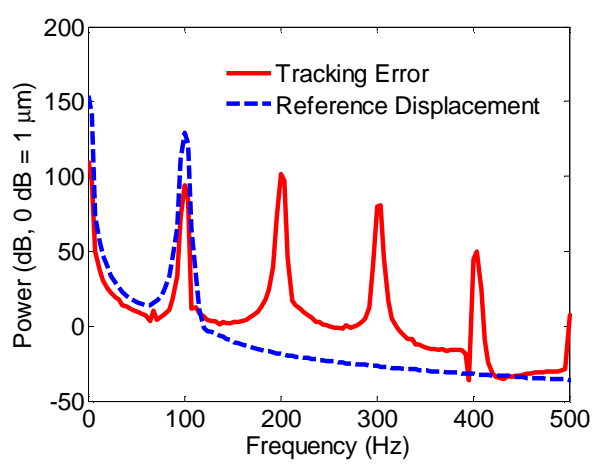

(b)

Figure 5: Nonlinear open loop tracking control performance in the presence of model uncertainty; (a) tracking performance in the time domain and (b) tracking performance and error in the frequency domain.

Tracking errors in the presence of model uncertainties were reduced by designing a narrowband filter using the control gains computed using (21). This requires solving an algebraic Ricatti equation (ARE) for a given set of control parameters $Q$ and $R$ in the cost function given by (20). The values of $\boldsymbol{Q}$ and $R$ were increased and decreased, respectively, to reduce RMS tracking error; see the Appendix for the values used. The narrowband controller was combined with integral control using a integral gain of $\mathrm{K}_{\mathrm{I}}=1 \times 10^{6}$ for all simulations. A fixed value for $R$ was chosen and penalties on each harmonic defined in the matrix $\boldsymbol{Q}$ were monotonically increased until the RMS error reached a minimum. This same process was repeated for each consecutive harmonic considered in the filter with the lower order penalties on each harmonic held fixed. The matrix components in $\boldsymbol{Q}$ were adjusted to penalize up to seven harmonics starting with a single harmonic at the reference displacement frequency and then the number of harmonics were incrementally increased at integer multiples of the reference displacement frequency. A similar procedure is used in the experimental implementation described in the following section.

The narrowband filter was combined with the nonlinear optimal control design to quantify performance enhancements in the presence of model uncertainties. The RMS and maximum percent tracking error are given in Table 2 as a function of the number of harmonics in the narrowband filter. Whereas the RMS error continuously decreases, only marginal reductions are observed for harmonics above $300 \mathrm{~Hz}$. In addition, the maximum tracking error reaches a minimum using the first three harmonics and then begins to increase as the number of harmonics increases.

To further illustrate control performance, tracking control simulations using the hybrid nonlinear optimal control design with narrowband perturbation feedback are plotted for the narrowband filter design using the first harmonic $(100 \mathrm{~Hz})$, the first three harmonics $(100 \mathrm{~Hz}-$ 
$300 \mathrm{~Hz}$ ) and all seven harmonics $(100 \mathrm{~Hz}-700 \mathrm{~Hz})$. The simulations are given in both the time domain and frequency domain in Figure 6. All of the frequency domain plots have been normalized using $0 \mathrm{~dB}$ as a $1 \mu \mathrm{m}$ reference displacement. The maximum tracking error in the steady state regime occurs when the narrowband filter only penalizes the $100 \mathrm{~Hz}$ reference displacement frequency. As additional harmonics up to $300 \mathrm{~Hz}$ are added to the narrowband filter, the tracking error harmonics are reduced. However, only small improvements are observed when more that the first three harmonics are included in the narrowband filter, in agreement with the results in Table 2. It should also be noted that certain higher harmonic tracking errors increase when the $100 \mathrm{~Hz}-700 \mathrm{~Hz}$ narrowband filter was used.

Table 2: RMS and maximum tracking errors over the entire simulation time. The narrowband filter harmonics start with one harmonic at $100 \mathrm{~Hz}$ and each addition harmonic is added to the filter with the filter gains at lower harmonics held fixed. The RMS and maximum tracking errors are normalized relative to the maximum reference displacement.

\begin{tabular}{ccc} 
Narrowband Filter & RMS Tracking Error $(\%)$ & Max Tracking Error $(\%)$ \\
\hline $0 \mathrm{~Hz}$ (open loop) & $7.00 \%$ & $7.11 \%$ \\
$100 \mathrm{~Hz}$ & $2.67 \%$ & $7.12 \%$ \\
$200 \mathrm{~Hz}$ & $2.45 \%$ & $6.59 \%$ \\
$300 \mathrm{~Hz}$ & $2.12 \%$ & $6.57 \%$ \\
$400 \mathrm{~Hz}$ & $2.03 \%$ & $7.17 \%$ \\
$500 \mathrm{~Hz}$ & $2.02 \%$ & $7.88 \%$ \\
$600 \mathrm{~Hz}$ & $2.01 \%$ & $7.99 \%$ \\
$700 \mathrm{~Hz}$ & $2.00 \%$ & $8.00 \%$
\end{tabular}

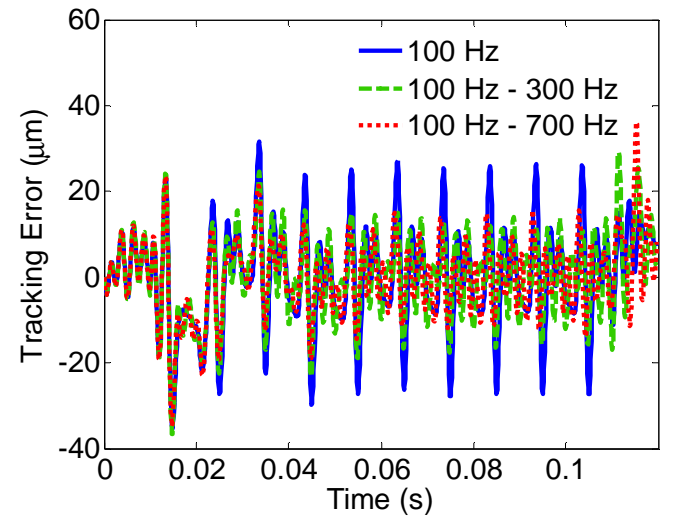

(a)

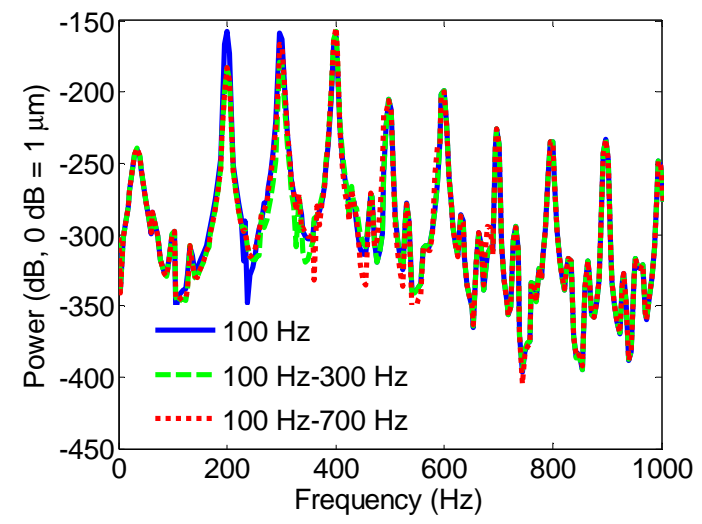

(b)

Figure 6: Narrowband control simulations for a $100 \mathrm{~Hz}$ reference frequency. An increasing number of higher-order harmonics are introduced in the narrowband filter. (a) Time domain tracking error. (b) Tracking error in the frequency domain using FFT. The FFT was computed in the steady state regime from $\mathrm{t}=0.03 \mathrm{sec}$ to $\mathrm{t}=0.11 \mathrm{sec}$.

For comparison to the nonlinear optimal control design, tracking control using only narrowband-integral feedback is simulated. The results illustrate slower transient responses 
relative to nonlinear control, but improved steady-state error reduction. Similar results are obtained with only one harmonic at $100 \mathrm{~Hz}$ and higher-order harmonics. The performance attributes of open loop control, hybrid control using nonlinear optimal control with narrowband perturbation feedback, and only narrowband feedback are plotted in Figure 7. The narrowband filter using $100 \mathrm{~Hz}-300 \mathrm{~Hz}$ was used in the simulations. These simulation results are compared with experimental results in the following section.

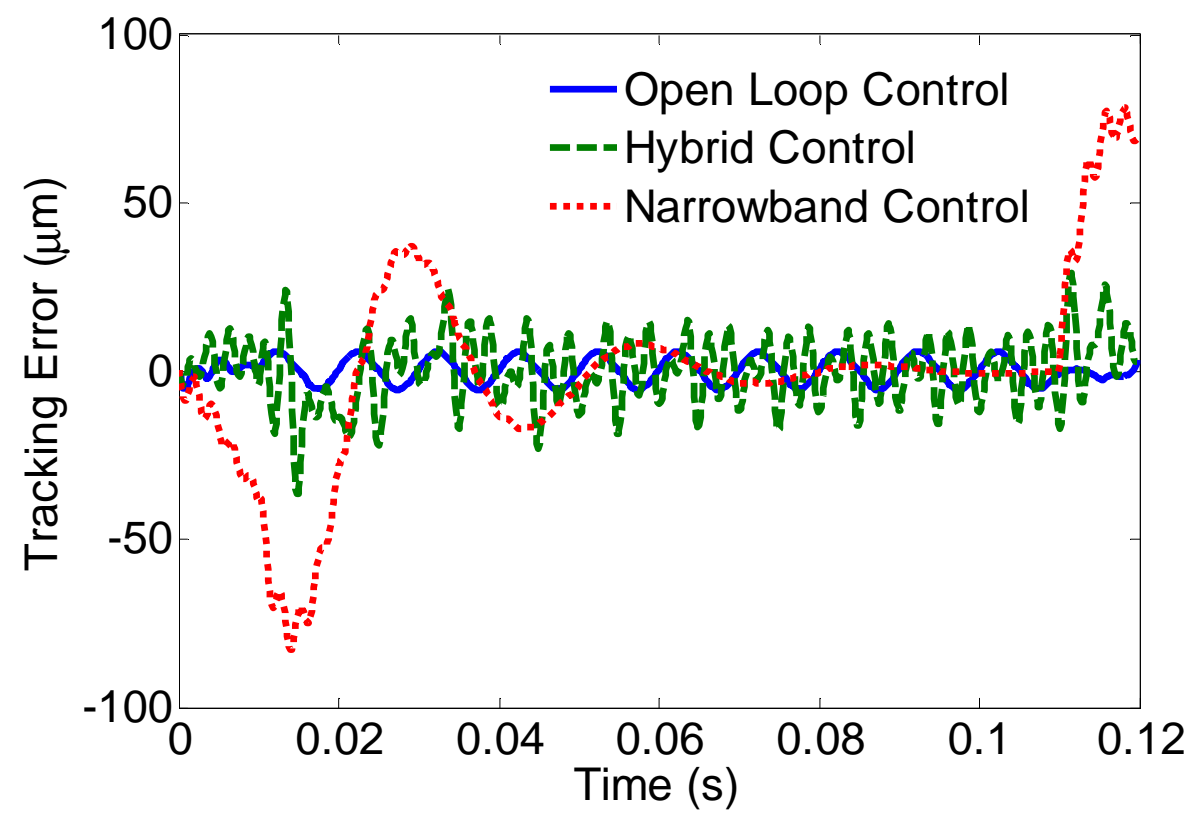

Figure 7: Tracking error comparison for each control design. The hybrid control design denotes nonlinear optimal control with narrowband perturbation feedback. In this case, the narrowband filter used the first three harmonics, $100 \mathrm{~Hz}-300 \mathrm{~Hz}$.

\section{Experimental Implementation}

The nonlinear optimal control design with narrowband-integral perturbation feedback is implemented experimentally and compared to the simulations. The reference displacements are tested for both a maximum reference displacement of $150 \mu \mathrm{m}$ and $450 \mu \mathrm{m}$. The reference displacement profile used in the experiments was the same as the simulations given in Section IV.3. Tracking errors are quantified using nonlinear open loop control, narrowband feedback, and combined nonlinear open loop control with narrowband perturbation feedback.

The experimental implementation of these control algorithms was accomplished using a magnetostrictive actuator with embedded displacement sensor, analog to digital conversion board, floating point processor board, digital to analog conversion board and a linear power amplifier. 
Specifically used were an Etrema Active Machining System actuator, part number PP12102833, dSPACE ds2003 (ADC board), ds1003 (processor board), ds2101 (DA board), and a AE Techron model 7780 linear power amplifier. The displacement sensor was a Lucas-Varity model 025MHR-006 LVDT. Additionally, a TRW Schaevitz LVM-110 signal conditioning module was used to interface the LVDT with the ADC board. The power amplifier was configured for controlled current mode with a gain of 4 amperes per volt. LVDT sensitivity was -70 um per volt.

The control algorithm and reference displacement signals were programmed into the processor board using Simulink and a block library available from dSPACE. Sampling occurred at $5 \mathrm{kHz}$ and both the ADC and DA boards used 16 bit converters. The processor board was used to both implement the closed loop control algorithms and log data to memory for post experiment analysis. The block diagram illustrating the experimental set-up is shown in Figure 8.

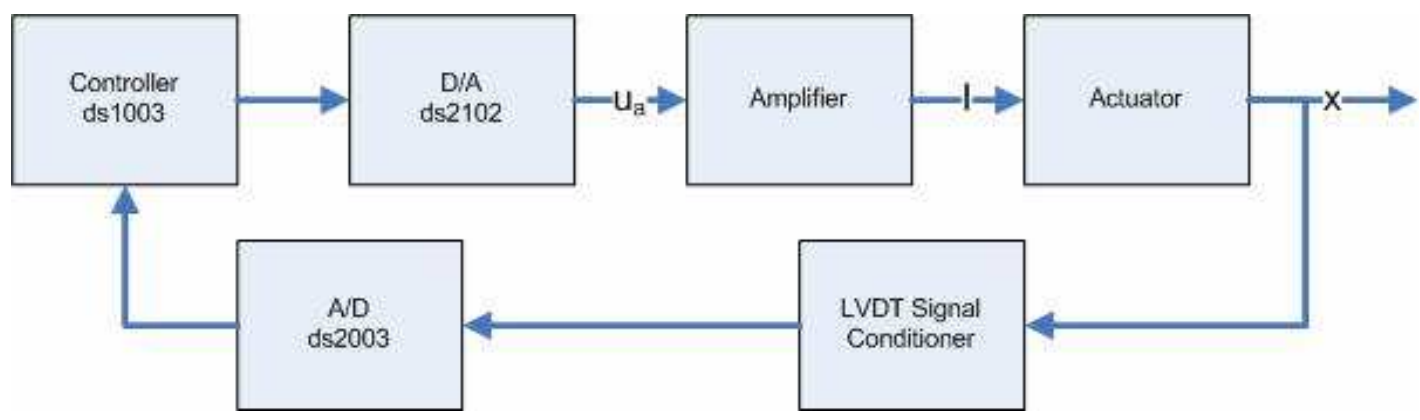

Figure 8: Block diagram of the experimental set-up used for control experiments and post experiment analysis.

Open loop tracking errors and the corresponding hysteresis for the two displacement levels are illustrated in Figure 9 which shows increases in model uncertainty at higher reference displacements. This is in agreement with the model fits in Figure 2 which contain a maximum of $80 \mu \mathrm{m}$ in error between the data and model near a field of $70 \mathrm{kA} / \mathrm{m}$ as this field is increased. The effect of this model uncertainty is compared with the feedback control design and perturbation feedback to identify how narrowband feedback and open loop nonlinear control affects tracking performance. 


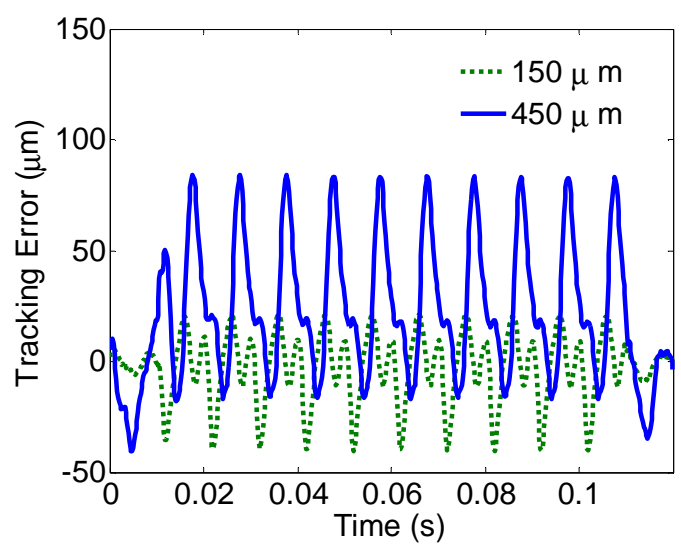

(a)

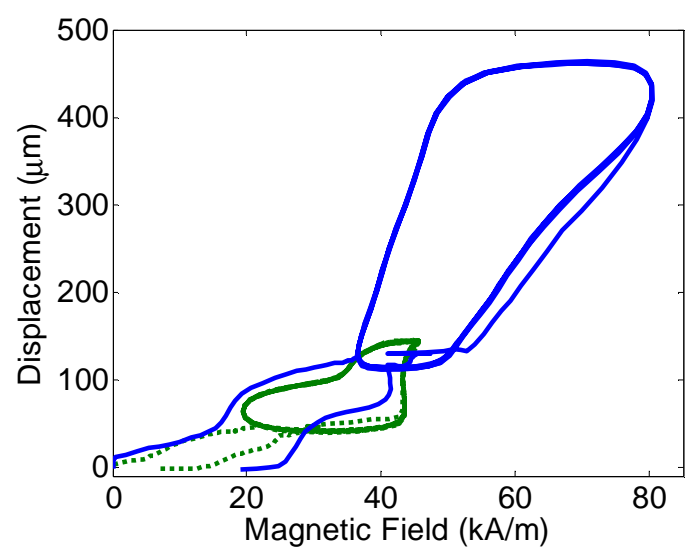

(b)

Figure 9: (a) Nonlinear open loop tracking error for reference displacements with a maximum of $150 \mu \mathrm{m}$ and $450 \mu \mathrm{m}$ and (b) the corresponding hysteresis behavior.

Time domain experimental results of the narrowband-integral control design and hybrid nonlinear optimal control with narrowband-integral perturbation feedback are illustrated in Figures 10 and 11; respectively. Gains for each harmonic were determined through experiments and include a degree of stability margin learned through trial and error. Tuning was conducted such that at a particular harmonic, the gain was increased until further increases yielded no additional improvement in tracking performance or caused system instability. In Figure 10, reasonable control is achieved in the steady-state oscillating regime while poor transient response is achieved. In Figure 11, the addition of open-loop control provides enhancements in transient behavior with a trade-off in increased steady-state error.

The RMS tracking error and maximum tracking error percentages are summarized in Table 3. The maximum tracking error percentages were calculated over the entire reference displacement signal while the RMS tracking error was calculated in the steady state regime. The percent errors show improvements in tracking control as the number of harmonics in the narrowband filter is increased for the $450 \mu \mathrm{m}$ reference displacement. However, slight increases in RMS error occur for the $150 \mu \mathrm{m}$ reference displacement when all seven harmonics are used in the filter relative to the filter that used only the first three harmonics. The maximum error is significantly reduced when the hybrid nonlinear optimal control with perturbation narrowband feedback is used relative to only using the narrowband filter. 


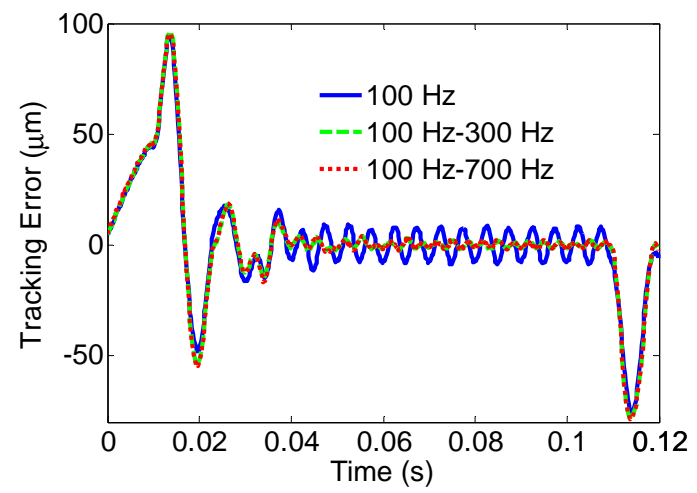

(a)

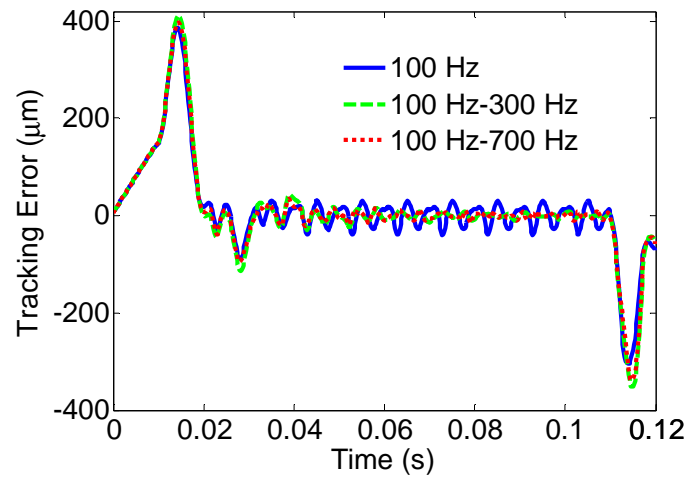

(b)

Figure 10: Tracking errors using only narrowband feedback. (a) Tracking control for the $150 \mu \mathrm{m}$ displacement using multiple harmonics, and (b) tracking error for the $450 \mu \mathrm{m}$ displacement using multiple harmonics.

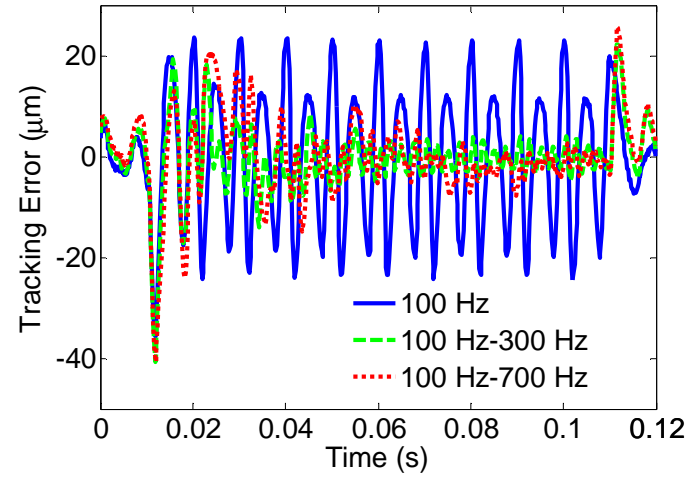

(a)

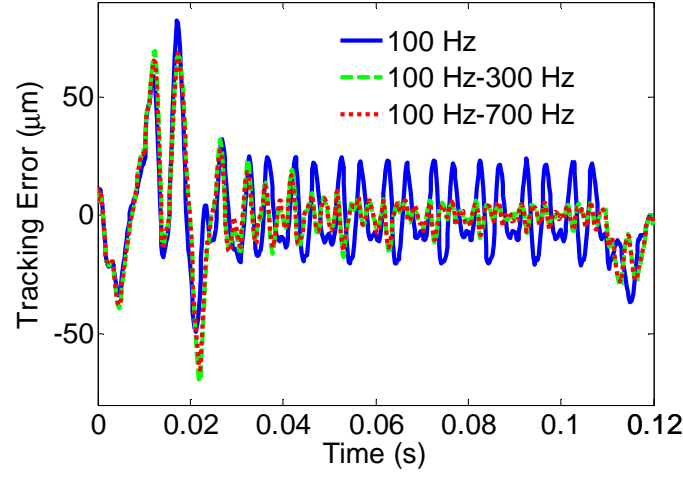

(b)

Figure 11: Tracking errors using nonlinear optimal control with narrowband perturbation feedback. (a) Tracking control for the $150 \mu \mathrm{m}$ displacement using multiple harmonics, and (b) tracking error for the 450 $\mu \mathrm{m}$ displacement using multiple harmonics.

Table 3: RMS and maximum percent tracking errors for narrowband control only and the hybrid control (nonlinear optimal control with narrowband feedback). *Note that the RMS error is only applied in the steady state regime.

\begin{tabular}{|cccccc|}
\hline & & \multicolumn{2}{c}{ Narrowband Control } & \multicolumn{2}{c|}{ Hybrid Control } \\
Filter Harmonics & Max Displacement & RMS Error* & Max Error & RMS Error* & Max Error \\
\hline $100 \mathrm{~Hz}$ & $150 \mu \mathrm{m}$ & $3.58 \%$ & $57.7 \%$ & $10.1 \%$ & $17.2 \%$ \\
$100 \mathrm{~Hz}-300 \mathrm{~Hz}$ & & $0.85 \%$ & $59.5 \%$ & $1.59 \%$ & $13.9 \%$ \\
$100 \mathrm{~Hz}-700 \mathrm{~Hz}$ & & $0.88 \%$ & $58.0 \%$ & $2.33 \%$ & $16.2 \%$ \\
\hline $100 \mathrm{~Hz}$ & $450 \mu \mathrm{m}$ & $4.40 \%$ & $89.8 \%$ & $3.01 \%$ & $17.6 \%$ \\
$100 \mathrm{~Hz}-300 \mathrm{~Hz}$ & & $1.43 \%$ & $91.3 \%$ & $1.02 \%$ & $15.1 \%$ \\
$100 \mathrm{~Hz}-700 \mathrm{~Hz}$ & & $1.34 \%$ & $88.0 \%$ & $0.97 \%$ & $15.0 \%$ \\
\hline
\end{tabular}




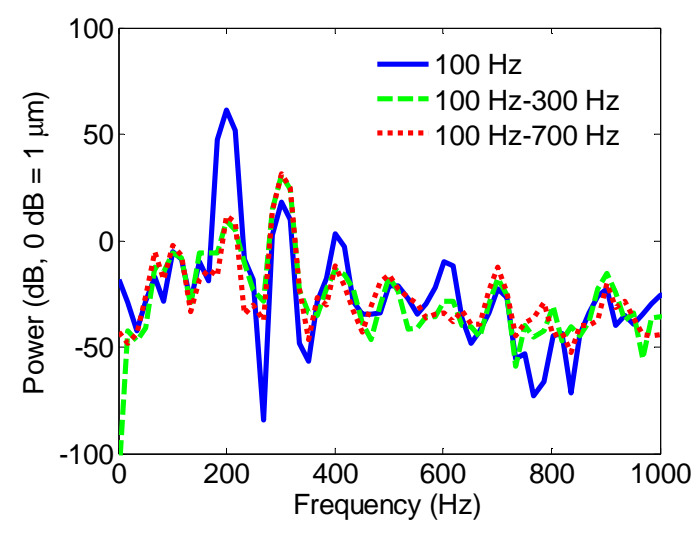

(a)

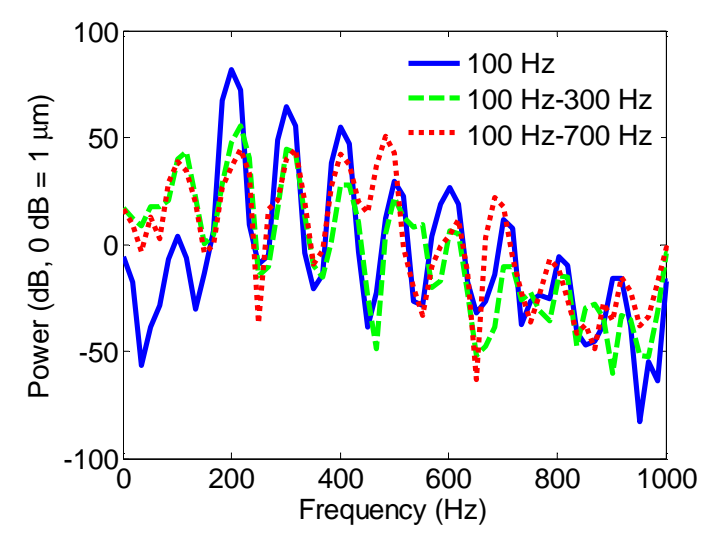

(b)

Figure 12: Tracking error in the frequency domain for the narrowband feedback control without nonlinear optimal control. (a) $150 \mu \mathrm{m}$ reference displacement and (b) $450 \mu \mathrm{m}$ reference displacement.

The fast Fourier transform of the data in Figures 10 and 11 is illustrated in Figures 12 and 13; respectively. These results are based on tracking errors in the steady-state oscillating regime. The FFT plots for each maximum displacement are denoted by $0 \mathrm{~dB}$ at $1 \mu \mathrm{m}$ tracking error. Significant reductions in tracking error are shown when one to three harmonics are included in the narrowband filter while additional reductions using seven harmonics are only minor. Certain tracking error harmonics increase when additional harmonics are added to the narrowband filter. The narrowband filter with only the $100 \mathrm{~Hz}$ filter provided significant reductions at this particularly frequency while in most cases, the higher-order filters provided reductions in certain limited bandwidths but reduced performance at other frequencies.

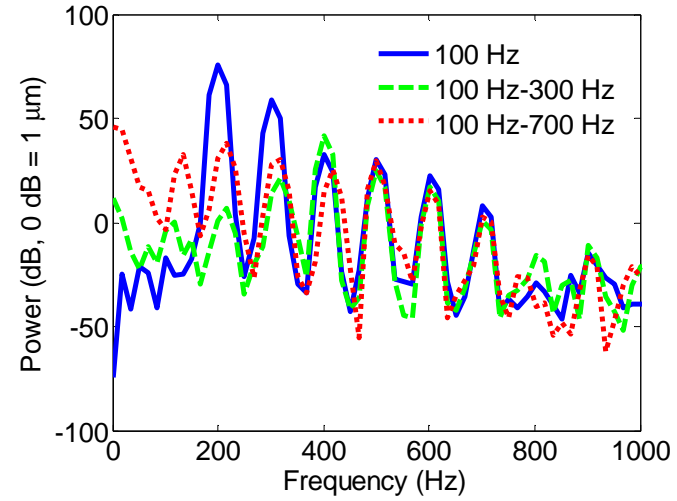

(a)

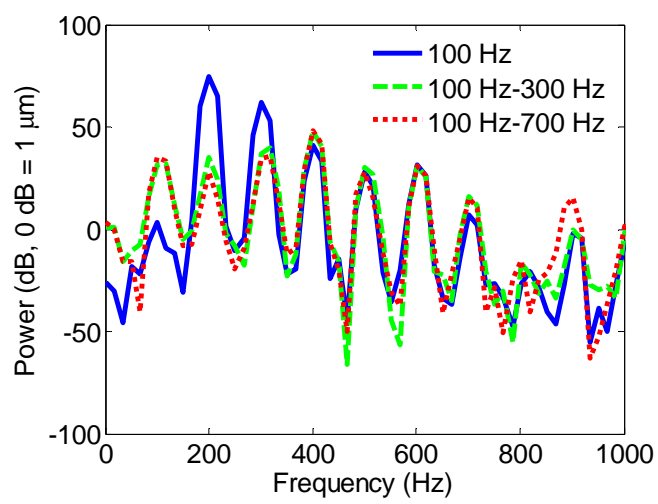

(b)

Figure 13: Tracking error in the frequency domain for the hybrid nonlinear optimal control design with narrowband perturbation feedback. (a) the $150 \mu \mathrm{m}$ reference displacement and (b) $450 \mu \mathrm{m}$ reference displacement. 


\section{Concluding Remarks}

A comparison of nonlinear open loop control and narrowband-integral feedback was presented to illustrate performance trade-offs when tracking a non-periodic reference displacement. An improvement in steady-state tracking is obtained using the narrowband filter whereas an enhancement in transient performance is obtained using nonlinear optimal control. The hybrid nonlinear control design that combines nonlinear optimal control with perturbation feedback shows overall improvements in tracking control; however, steady-state tracking errors are increased relative to only using narrowband feedback control. The simulations and control experiments both illustrate reductions in tracking error harmonics using a limited number of higher-order harmonics in the narrowband filter. This illustrates that a careful consideration of the gains and number of narrowband harmonics must be assessed to quantify trade-offs in tracking performance and potential excitation of unstable modes in the actuator.

The results suggest additional tuning using adaptive filters should be considered to optimize performance in both bandwidth and steady-state tracking. However, the results presented here are promising for high speed control problems since they illustrate a reduction in the amount of feedback necessary to achieve high bandwidth in a nonlinear operating regime for a number of smart structure applications that operate in the nonlinear and hysteretic regime.

\section{Acknowledgements}

The authors gratefully acknowledge support from ONR under contract N00014-05-C0165.

\section{Appendix}

The values used in the narrowband control filter and the nonlinear optimal control design are given here. A single set of cost function penalties for the nonlinear optimal control design were used which consist of $Q_{u}=1 \times 10^{17}$ and $Q_{v} \cong 0$ and $\mathrm{R}=1 \times 10^{-17}$ and are based on SI units. The subscript $u$ and $v$ denote penalties on the tracking error displacement and velocity, respectively.

The penalties used in the narrowband filter design are given in Table 4. For each harmonic, the penalty on $Q_{v}$ was set to one to provide damping and similarly the penalty on the control input

was $R=1 \times 10^{-7}$ for each harmonic. It should be noted that all higher-order frequency penalties on $Q_{u}$ were set to zero unless these harmonics were "activated" in the narrowband filter. All values used up to $700 \mathrm{~Hz}$ are given in Table 4. These penalties for each harmonic are combined with integral control using a gain of $\mathrm{K}_{\mathrm{I}}=1 \times 10^{6}$. 
The control gains used in the experiments are given for each narrowband filter design. These parameters are based on the notation given in (Sievers 1992) for higher harmonic control since this facilitates experimental implementation; however, the results can be directly related to optimal control parameters $Q$ and $R$ by converting the state space representation to the Laplace domain. The transfer function for the filter is

$$
H(s)=\frac{2 k\left(a s+b \omega_{0}\right)}{s^{2}+\omega_{0}^{2}}
$$

where $\mathrm{k}$ is a feedback gain, $\omega_{0}$ is the narrowband filter frequency. The "a" and "b" values are unique constants for each harmonic based on open loop plant dynamics. The parameter "a" is the real component of the plant transfer function evaluated at the harmonic frequency of interest. Similarly, "b" is the imaginary component of the plant transfer function at the harmonic frequency. The gains used in the experiments are given in Table 5.

Table 4: Cost function penalty values used in the narrowband filter design which were used in the simulations. Up to seven harmonics relative to the input tracking signal at $100 \mathrm{~Hz}$ were used.

\begin{tabular}{|c|c|}
\multicolumn{1}{c|}{ Frequency $(\mathrm{Hz})$} & $Q_{u}$ \\
\hline 100 & $2 \times 10^{3}$ \\
200 & $1 \times 10^{-1}$ \\
300 & $1 \times 10^{-2}$ \\
400 & $1 \times 10^{-4}$ \\
500 & $1 \times 10^{-4}$ \\
600 & $1 \times 10^{-4}$ \\
700 & $5 \times 10^{-5}$ \\
\hline
\end{tabular}

Table 5: Narrowband gains $k_{i}$ where 1) $i=1$ is for the $150 \mu \mathrm{m}$ reference displacement with no nonlinear optimal control, 2) $i=2$ is for the $150 \mu \mathrm{m}$ reference displacement with nonlinear optimal control, 3 ) $i=3$ is for the $450 \mu \mathrm{m}$ reference displacement with no nonlinear optimal control, and 4) $i=4$ is for the $450 \mu \mathrm{m}$ reference displacement with nonlinear optimal control.

\begin{tabular}{|c|c|c|c|c|}
\multicolumn{1}{c}{ Frequency $(\mathrm{Hz})$} & $\mathrm{k}_{1}$ & $\mathrm{k}_{2}$ & $\mathrm{k}_{3}$ & $\mathrm{k}_{4}$ \\
\hline Integrator & 1000 & 100 & 300 & 300 \\
100 & 5000 & 8000 & 2500 & 2000 \\
200 & 3000 & 6000 & 2500 & 2000 \\
300 & 500 & 6000 & 2500 & 3000 \\
400 & 100 & 8000 & 500 & 100 \\
500 & 100 & 20 & -5000 & -2000 \\
600 & 100 & 100 & 100 & 100 \\
700 & 100 & 100 & 100 & 100 \\
\hline
\end{tabular}




\section{References}

Bay, J. 1999. Fundamentals of Linear State Space Systems, WCB McGraw-Hill, Boston.

Cheng, R., Tischler, M., Celi, R. 2006. "A high-order, linear time-invariant model for application to higher harmonic control and flilght control system interaction." NASA Tech. Report, NASA/TP-2002006-213460: 1-175.

Clarke, D., Mohtadi, C., Tuffs, P. 1987. "Generalized Predictive Control--Part I. The Basic Algorithm." Automatica, 23(2): 137-148.

Croft, D., Shed, G., Devasia, S. 2001. "Creep, hysteresis, and vibration compensation for piezoactuators: Atomic force microscopy application." J. Dyn. Syst.-T. ASME, 23: 35-43.

Ge, P., Jouaneh, M. 1996. "Tracking control of a piezoceramic actuator." IEEE T. Contr. Syst. T., 4(3): 209-216.

Hall, S., Wereley, N. 1992. "Performance of Higher Harmonic Control Algorithms for Helicopter Vibration Reduction." J. Guidance, 16(4): 793-797.

Henson, M. 1998. "Nonlinear model predictive control: current status and future directions." Computers and Chemical Engineering, 23: 187-202.

Ikhouane, F., Rodeller, J. 2007. Systems with Hysteresis: Analysis, Identification and Control Using the Bouc-Wen Model, Wiley.

Iyer, R., Tan, X. 2009. "Control of hysteretic systems through inverse compensation: Inversion algorithms, adaptation, and embedded implementation." IEEEContr. Syst. Mag., 29(1): 83-99.

Iyer, R., Tan, X., Krishnaprasad, P. 2005. "Approximate inversion of the Preisach hysteresis operator with application to control of smart actuators." IEEE T. Automat. Contr., 50(6): 798-810.

Janocha, H., Kuhnen, K. 2000. "Real-time compensation of hysteresis and creep in piezoelectric actuators." Sensors and Actuators, 79: 83-89.

Janocha, H., Pesotski, D., Kuhnen, K. 2008. "FPGA-based compensator of hysteretic actuator nonlinearities for highly dynamic applications." IEEE/ASME T. Mech., 13(1): 112-116.

Kirkpatrick, S., Gelatt, C., Vecchi, M. 1983. "Optimization by Simulated Annealing." Science, 220(4598): 671-680.

Lewis, F., Syrmos, V. 1995. Optimal Control, Wiley-Interscience.

Lovera, M., Colaneri, P., Celi, R. 2003. "Periodic analysis of Higher Harmonic Control techniques for helicopter vibration attenuation." Proc. Am. Control Conf., Denver, CO June 4-6, 2003: 999-1004.

Mracek, C., Cloutier, J. 1998. "Control designs for the nonlinear benchmark problem via the state-dependent Riccati equation method." Int. J. Robust Nonlinear Contrl, 8: 401-433.

Natale, C., Velardi, F., Visone, C. 2001. "Modelling and compensation of hysteresis for magnetostrictive actuators." in 2001 IEEE/ASME Int.Conf. Adv. Intell. Mechatron., Como, Italy: 444-479.

Oates, W., Evans, P., Smith, R., Dapino, M. 2009a. "Experimental implementation of a hybrid nonlinear control design for magnetostrictive transducers." J. Dyn. Syst.-T. ASME, 131(3): 041004-041015. 
Oates, W., Smith, R. 2009b. "Optimal tracking using magnetostrictive actuators operating in the nonlinear and hysteretic regime." J. Dyn. Syst.-T. ASME, 131(3): 031001031012.

Sievers, L., von Flotow, A. 1992. "Comparison and Extensions of Control Methods for Narrow-Band Distrubance Rejection." IEEE T. Signal Proces., 40(10).

Smith, R. 2005. Smart Material Systems: Model Development, SIAM, Philadelphia.

Smith, R., Seelecke, S., Dapino, M., Ounaies, A. 2006. "A unified framework for modeling hysteresis in ferroic materials." J. Mech. Phys. Solids, 54(1): 46-85.

Tan, X., Baras, J. 2004. "Modeling and control of hysteresis in magnetostrictive actuators." Automatica, 40(9): 1469-1480.

Tian, Y., Song, Q., Cattafesta, L. 2006. "Adaptive Feedback Control of Flow Separation." 3rd AIAA Flow Control Conference, AIAA 2006-3016: 1-11. 